\title{
Beyond free radical scavenging: Beneficial effects of edaravone (Radicut) in various diseases (Review)
}

\author{
KIYOSHI KIKUCHI $^{1,2 *}$, NOBUYUKI TAKESHIGE ${ }^{2 *}$, NAOKI MIURA $^{3 *}$, YOKO MORIMOTO $^{4}$, \\ TAKASHI ITO $^{5}$, SALUNYA TANCHAROEN ${ }^{6}$, KEI MIYATA ${ }^{5}$, CHIEMI KIKUCHI $^{7}$, NARUMI IIDA ${ }^{8}$, \\ HISAAKI UCHIKADO ${ }^{2}$, NAOHISA MIYAGI ${ }^{1}$, NAOTO SHIOMI ${ }^{9}$, TERUKAZU KURAMOTO ${ }^{10}$, \\ IKURO MARUYAMA ${ }^{5}$, MOTOHIRO MORIOKA ${ }^{2}$ and KO-ICHI KAWAHARA ${ }^{5,11}$
}

\begin{abstract}
${ }^{1}$ Department of Neurosurgery, Yame Public General Hospital, Yame 834-0034; ${ }^{2}$ Department of Neurosurgery, Kurume University School of Medicine, Kurume 830-0011; ${ }^{3}$ Veterinary Teaching Hospital and Laboratory of Veterinary Diagnostic Imaging, Faculty of Agriculture, Kagoshima University, Kagoshima 890-0065; ${ }^{4}$ Department of Restorative Dentistry and Endodontology, Kagoshima University Graduate School of Medical and Dental Sciences, Kagoshima 890-8544;

${ }^{5}$ Systems Biology Thrombogenesis Regulation, Kagoshima University Graduate School of Medical and Dental Sciences, Kagoshima 890-8520, Japan; ${ }^{6}$ Department of Pharmacology, Faculty of Dentistry, Mahidol University, Rajthevee, Bangkok 10400, Thailand; ${ }^{7}$ Department of Rehabilitation, Nishida Koutoku Hospital, Oita 876-0047; ${ }^{8}$ Research Laboratory, Kohjin Co., Ltd., Oita 876-8580; ${ }^{9}$ Department of Emergency, Saiseikai Shiga Hospital, Shiga 520-3046;

${ }^{10}$ Department of Neurosurgery, Omuta City General Hospital, Fukuoka 836-8567; ${ }^{11}$ Laboratory of Functional Foods, Department of Biomedical Engineering Osaka Institute of Technology, Osaka 535-8585, Japan
\end{abstract}

Received July 22, 2011; Accepted September 13, 2011

DOI: $10.3892 / \mathrm{etm} .2011 .352$

\begin{abstract}
Free radicals play an important role in the pathogenesis of a variety of diseases; thus, they are an attractive target for therapeutic intervention in these diseases. Compounds capable of scavenging free radicals have been developed for this purpose and some, developed for the treatment of cerebral ischemic stroke, have progressed to clinical trials. One such scavenger, edaravone, is used to treat patients within $24 \mathrm{~h}$ of stroke. Edaravone, which can diffuse into many disease-affected organs, also shows protective effects in the heart, lung, intestine, liver, pancreas, kidney, bladder and testis. As well as scavenging free radicals, edaravone has antiapoptotic, anti-necrotic and anti-cytokine effects in various diseases. Here, we critically review the literature on its clinical efficacy and examine whether edaravone should be considered a candidate for worldwide development, focusing on its effects on diseases other than cerebral infarction. Edaravone has been safely used as a free radical scavenger for more than 10 years;
\end{abstract}

Correspondence to: Dr Ko-ichi Kawahara, Systems Biology Thrombogenesis Regulation, Kagoshima University Graduate School of Medical and Dental Sciences, 8-35-1 Sakuragaoka, Kagoshima 890-8520, Japan

E-mail: telo@m3.kufm.kagoshima-u.ac.jp

*Contributed equally

Key words: cerebral infarction, edaravone, Radicut, free radical scavenger, non-neurologic disease we propose that edaravone may offer a novel treatment option for several diseases.

\section{Contents}

1. Introduction

2. Pharmacological effects of edaravone in non-neurologic diseases

3. Conclusion

\section{Introduction}

Many compounds have been evaluated as free radical scavengers for the treatment of cerebral ischemic stroke, but few have been successful in studies conducted in Western countries. By contrast, trials conducted by Japanese researchers have been more successful (1). Several free radical scavengers have been developed and several of these (e.g., ebselen, tirilazad and NXY-059) have progressed to clinical trials (2). Ebselen and tirilazad produced inadequate therapeutic effects in patients with cerebral infarction and further studies were terminated $(3,4)$, while the Stroke-Acute Ischemic NXY Treatment II trial showed that NXY-059 was not effective against cerebral infarction when administered within $4 \mathrm{~h}$ of onset of symptoms (5).

Edaravone (3-methyl-1-phenyl-2-pyrazolin-5-one, MCI-186, Radicut; Mitsubishi Tanabe Pharma Corporation, Osaka, Japan) was the first neuroprotective drug to be introduced worldwide. Since 2001, it has been used in Japan to treat many patients with cerebral ischemic stroke (6-8). It is 
currently approved only in Japan. The free radical scavenger edaravone exerts anti-oxidant effects by inhibiting hydroxyl radical-dependent and independent lipid peroxidation $(7,9)$. This anti-oxidant activity, which is proposed to be the main mechanism of action of edaravone, may protect against free radical-related injuries following cerebral ischemic stroke (10). Edaravone was also shown to suppress increases in hydroxyl and superoxide anion radical levels in several models of cerebral ischemic stroke $(11,12)$.

Edaravone exerts effects that are unrelated to its free radical scavenging actions and that may be useful in the treatment of diseases other than cerebral infarction. To the best of our knowledge, potential use of edaravone in the treatment of other diseases has not been reviewed elsewhere. Therefore, in the present review, we discuss the most recent research on the use of edaravone, primarily in animal models of various diseases. We also assess its potential use in the treatment of these diseases.

\section{Pharmacological effects of edaravone in non-neurologic diseases}

Effects of edaravone on apoptosis. Anti-apoptotic effects of edaravone have been reported in studies of several diseases, including non-neurologic diseases. In a rat coronary occlusion model, edaravone reduced the myocardial infarction area, maintained myocardial ATP content, decreased mitochondrial swelling, reduced cytochrome c release, increased the expression of $\mathrm{BCl}-2$ and reduced the number of apoptotic cells and DNA fragmentation (13). Edaravone ameliorated the progression of experimental autoimmune myocarditis (EAM), it improved left ventricular (LV) function and decreased the number of TUNEL-positive cells in the LV of rats with EAM (14). Edaravone attenuated apoptotic cell death in rabbits with bleomycin-induced pulmonary injury (15).

Edaravone blunted ischemia/reperfusion (I/R)-induced hepatic dysfunction and hepatic apoptosis in rats (16). Edaravone treatment blunted the carbon tetrachloride $\left(\mathrm{CCL}_{4}\right)$-induced elevation in serum alanine aminotransferase (ALT), serum lactate dehydrogenase (LDH), serum total bilirubin, hepatic fatty degeneration, hepatic steatosis and hepatic apoptosis in rats $(17,18)$. In vitro, edaravone appears to protect hepatocytes from Fas-induced mitochondrial-dependent apoptosis by regulating mitochondrial $\mathrm{Bcl}-\mathrm{xL}$ and $\mathrm{Bax}$ in mice with fulminant hepatic failure (19).

In streptozotocin-induced diabetic rats, edaravone promoted engraftment of intraportally transplanted islet cells, ameliorated hyperglycemia, increased insulin secretion and the number and size of islet $\beta$ cells morphologically and decreased the number of TUNEL-positive cells in each islet (20).

Edaravone attenuated cisplatin-induced renal dysfunction, renal tubular damage, mitochondrial damage, real protein oxidation and tubular apoptosis in rats (21).

Edaravone also suppressed X-ray-induced apoptosis in the human T-cell leukemia cell line, MOLT-4 $(22,23)$.

Effects of edaravone on necrosis. Anti-necrotic effects of edaravone have been reported in studies of multiple diseases, including non-neurologic diseases. For example, edaravone reduced the area of necrotic myocardium in rats (24) and rabbits (25) in myocardial I/R models. Edaravone blunted the I/R-induced elevation in hepatic dysfunction and hepatic necrosis in rats $(16,26)$. Edaravone treatment also blunted $\mathrm{CCL}_{4}$-induced hepatic necrosis in rats (17). Edaravone treatment blunted the I/R-induced elevation in serum ALT, hepatic congestion, hepatic vacuolization and hepatic necrosis in rats (27).

In a rat sodium taurocholate-induced pancreatitis model, edaravone reduced plasma amylase levels, pancreatic myeloperoxidase (MPO; an indicator of neutrophil infiltration) activity, necrosis, edema and inflammatory cell infiltration (28).

Edaravone reversed cisplatin-induced renal dysfunction in rats, including an elevation in blood urea nitrogen and creatinine and histological changes, such as vacuolization, necrosis and protein casts (29). Edaravone attenuated renal dysfunction and acute tubular necrosis in a rat I/R acute renal failure (ARF) model (30).

Edaravone also reduced cell swelling, tubular vacuolization and necrosis in rat testis with I/R injury (31).

Effects of edaravone on oxidative stress. Anti-oxidant effects of edaravone have been reported in studies of multiple diseases, including non-neurologic diseases. Edaravone decreased serum concentrations of creatine kinase-MB isoenzymes, attenuated infarct size and improved ventricular ejection in 80 patients with acute myocardial infarction (AMI) (32). Edaravone attenuated infarct size, reperfusion arrhythmia and serum thioredoxin (a marker of oxidative stress) in 101 patients with AMI (33). Edaravone preserved coronary microvascular endothelial function, increased nitric oxide (NO) and decreased ROS in dogs with I/R injury (34). Edaravone significantly reduced MI size and improved cardiac function and LV remodeling by decreasing hydroxyl radicals and superoxide in the myocardium and increasing the production of NO during reperfusion in rabbits (35). Furthermore, in rats, edaravone prevented lethal reperfusion ventricular tachyarrhythmias and deteriorated cardiac function with ischemia and I/R injuries through inhibition of lipid peroxidation by scavenging free radicals (36). The addition of edaravone to cardioplegic solution ameliorated myocardial functional impairment by reducing oxidative stress after cardioplegic arrest in rats as well (37). Edaravone reduced myocardial oxidative stress overload with DNA damage, decreased myocardial protein carbonyl contents, the myocardial thiobarbituric acid reactive substance products, the formation of hydroxyl radicals and cytotoxic activities of lymphocytes in rats with EAM. It protected against acute EAM in rats by scavenging hydroxyl free radicals, resulting in the suppression of autoimmune-mediated myocardial damage associated with a reduced oxidative stress state (38). Edaravone ameliorated the progression of EAM in rats, improved LV function and decreased LV expression of nicotinamide adenine dinucleotide phosphate oxidase (NADPH) sub-unit [p67 (phox)] (14). Edaravone also exerted a cardioprotective action by inhibiting lipid peroxidation in a pig heart transplantation model (39). In an in vitro study, edaravone reduced I/R-induced cell death by attenuating ROS production in rabbit cardiomyocytes (40).

Edaravone blunted I/R-induced pulmonary dysfunction, pulmonary focal hyaline membrane formation, pulmonary neutrophil infiltration, pulmonary interstitial edema and oxidative stress markers, such as malondialdehyde (MDA), in 
dogs (41). Edaravone improved the survival rate of I/R-induced rabbits, reduced the production of hydroxyl radicals and MDA, increased the activities of glutathione peroxidase and superoxide dismutase (SOD) and reduced mitochondrial damage in lung tissue (42). In an isolated rat lung model, edaravone blunted the I/R-induced elevation in pulmonary dysfunction by suppressing pulmonary MDA and MPO activity, phospholipase (PL) $\mathrm{A}_{2}$ activation, which otherwise partially mediated edema formation, and neutrophil extravasation mediated by platelet-activating factor (PAF) receptor (43). In mice with bleomycin-induced pulmonary injury, edaravone improved the survival rate, reduced fibrotic change and the production of lipid hydroperoxide (LPO) in bronchoalveolar lavage fluid (BALF) and serum and increased the production of prostaglandin $\mathrm{E}_{2}$ in BALF (44).

Edaravone reduced I/R-induced small intestine injury, the levels of intraluminal protein and hemoglobin (an index of mucosal injury), thiobarbituric acid-reactive substances (TBARS; indicators of lipid peroxidation), tissue-associated MPO activity and multiple erosions and bleeding in rats (45). In a rat model of the acute phase of Crohn's disease, edaravone reduced the ulcer index, histological damage score, markers of oxidative damage, such as MPO activity and TBARS concentration, and ameliorated mesenteric indomethacin-induced longitudinal ulceration of the small intestine (46).

Edaravone improved the survival rate of LPS-treated rats and inhibited the increase in serum ALT levels and 4-hydroxynonenal (HNE)-modified proteins in rat liver tissue (47). Edaravone markedly improved the survival rate of I/R-induced liver injury in rats and decreased the levels of serum aspartate transaminase (AST), serum ALT and MPO activity (48). Similarly, edaravone treatment blunted I/R-induced elevation in serum ALT, serum hyaluronic acid (HA), lipid peroxidation and free radicals in rat liver (49). Edaravone ameliorated I/R-induced hepatic dysfunction and lipid peroxidation of perfusate in rats (50). Edaravone protects against mitochondrial injury, which prevents mitochondrial lipid peroxidation, inhibits the decrease in glutathione activity and improves I/R-induced hepatic energy metabolism in rats (51). Edaravone blunted I/R-induced hepatic dysfunction and an increase in MDA levels in rats (16). Furthermore, in rats, edaravone reduced hepatic I/R injury by minimizing hepatic lipid peroxidation (52). Edaravone treatment blunted $\mathrm{CCL}_{4}$-induced increases in the levels of oxidative stress markers, such as MDA, 4-HNE and 8-hydroxydeoxyguanosine $(\mathrm{OHdG})$, in rats with hepatic injury $(17,26)$. Edaravone treatment was also shown to blunt I/R-induced elevation in hepatic lipid peroxidation in rats (27). In another study, edaravone prevented LPS-induced liver injury after partial hepatectomy by attenuating oxidative damage and by reducing the production of MDA, NO and iNOS in rats (53). In this study, edaravone markedly improved the survival rate of LPS-treated rats after hepatectomy and inhibited increases in serum AST and LDH (53). Histopathological analysis revealed that edaravone also prevented inflammatory changes in the liver, kidney and spleen (53). An in vitro study involving primary cultures of rat hepatocytes further revealed that edaravone directly inhibits the induction of iNOS gene expression at the steps of its promoter transactivation and mRNA stabilization in IL-1 $\beta$-stimulated hepatocytes (54).
In a rat closed duodenal loop (CDL)-induced pancreatitis model, edaravone treatment tended to reduce ascites volume and to inhibit increases in wet pancreatic weight (55). Edaravone also tended to reduce microscopic mucosal damage scores and pancreatic tissue lipid peroxide levels (55).

Edaravone protected renal tubular epithelial cells and vascular endothelial cells, ameliorated renal dysfunction and inhibited increases in the levels of MDA and 8-OHdG in a canine I/R injury model (56). In a rat I/R ARF model, edaravone attenuated renal dysfunction, ROS production and lipid peroxidation (30). Edaravone was able to delay and improve urinary protein excretion in accordance with urinary $8-\mathrm{OHdG}$ excretion in puromycin nephrosis in rats (57). Edaravone reduced urinary protein and the levels of glomerular TBARS in rats with puromycin nephrosis (58). An in vitro study revealed that edaravone attenuated cisplatin-induced cell death, mitochondrial transmembrane potential loss and ROS production in murine proximal tubular cells (21).

Edaravone protected the contractile response during both field stimulation and carbachol exposure and reduced MDA levels after I/R-induced damage in the rat bladder (59). Acute urinary retention (AUR) and subsequent catheterization caused lipid peroxidation and oxidative DNA damage in the rat bladder (60). Edaravone induced a decrease in blood flow in the bladder during urinary retention and subsequent catheterization (60). Edaravone protected the contractile responses to both carbachol and $\mathrm{KCl}$ and reduced the levels of MDA and 8-OHdG after AUR and subsequent catheterization-induced bladder dysfunction in rats (60).

Similarly, in an I/R-induced rat testicular torsion model, edaravone reduced $\mathrm{NO}_{2}-\mathrm{NO}_{3}$ (a marker of $\mathrm{NO}$ production), MDA, 8-OHdG and MPO (31).

Edaravone suppressed free radicals (e.g. NO and total hydroperoxide), thereby maintaining mean arterial pressure (MAP) and prolonging survival time in a neonatal sepsis cecal ligation and perforation (CLP) model in piglets (61).

Edaravone treatment significantly reduced the levels of free radical precursors, such as MDA and xanthine oxidase, and their metabolites in the serum and tissue compared to controls in burn rats (62).

Edaravone diminished intestinal neutrophil lipid peroxidation and bacterial translocation in a rat hemorrhagic shock (HS) model (63). Furthermore, edaravone improved the survival rate in a rat HS model without resuscitation (64).

The administration of high doses of methamphetamine causes the degeneration of striatal dopaminergic fibers in the brains of rodents, and oxidative stress appears to be one of the main neurotoxic factors (65). Edaravone protected against methamphetamine-induced neurotoxicity in the striatum by blocking peroxynitrite production in mice (65). Edaravone blocked the increase in 3-nitrotyrosine (a biomarker of ROS) immunoreactivity and the activation of astrocytes (65).

Effects of edaravone on cytokines. Anti-cytokine effects of edaravone have been reported in studies of various nonneurologic diseases. Edaravone suppressed plasma monocyte chemoattractant protein-1 (MCP-1), improved left ventricular ejection fraction and reduced rehospitalization due to heart failure in 45 patients with AMI (66). Edaravone reduced myocardial IL-1 $\beta$-positive cells in rats with EAM (38). 
Edaravone protected cardiac function and reduced infarct size via a decrease in myocardial TNF- $\alpha$ production induced by $\mathrm{I} / \mathrm{R}$ injury in rats (67). Edaravone reduced the number of IL-1 $\beta$-positive cells in rats with acute EAM as well (68).

In the LPS-induced acute lung injury mouse model, edaravone prevented lung injury and attenuated inflammatory cells and pro-inflammatory cytokine production, such as IL-6, TNF- $\alpha$, keratinocyte-derived chemokine and macrophage inflammatory protein (MIP)-2 in BALF (69). Edaravone attenuated inflammatory cells, interstitial fibrosis, peribronchial fibrosis and transforming growth factor- $\beta$-positive cells in rabbits with bleomycin-induced pulmonary injury (15).

In the rat small intestine, edaravone reduced I/R injury and the levels of cytokine-induced neutrophil chemoattractant (CINC)-1 (a member of the IL- 8 family) protein and mRNA (45).

Edaravone prevented LPS-induced liver injury by both inhibition of inflammatory cell recruitment and expression of inflammatory cytokines in the rat liver (47). Moreover, mRNA expression levels of MIP-2, MCP-1 and MCP-5 were attenuated by edaravone (47). As a result, increases in the number of infiltrating inflammatory cells and mRNA expression of inflammatory cytokines, such as TNF- $\alpha$ and IL- 6 , were significantly blunted by edaravone in the rat liver (47). This reduction was accompanied by a significant reduction in their serum levels (47). Edaravone markedly improved the survival rate of I/R-induced liver injury and decreased the levels of serum AST, serum ALT and IL- 6 mRNA in rats (48). Edaravone treatment blunted I/R-induced elevation in serum ALT (49), serum HA (49), hepatic TNF- $\alpha$ mRNA (49), serum TNF- $\alpha$ (49), perfusate TNF- $\alpha$ (50), perfusate IL-1 $\beta$ (50), serum IL-6 (49), Kupffer cell TNF- $\alpha$ mRNA (49) and leukocyte infiltration (49) in the rat liver. Edaravone treatment blunted $\mathrm{CCL}_{4}$-induced elevation in serum IL-6, serum IL-10, serum TNF- $\alpha$, hepatic TNF- $\alpha$ mRNA, hepatic IL-4 mRNA, hepatic IL-6 mRNA and hepatic IL-10 mRNA in rats with hepatic injury (17). Edaravone blunted I/R-induced elevation in IL-10 of perfusate in rats (26). Edaravone treatment blunted I/R-induced elevation in hepatic tissue monocytes and neutrophils, hepatic IL-1 $\beta$, CINC-2, MIP-2, MCP-1, MIP-1 $\alpha$, MIP-1 $\alpha$ and intercellular adhesion molecule (ICAM)-1 mRNA in rats (27). In another study, edaravone prevented LPS-induced liver injury after partial hepatectomy by reducing the production of CINC and inflammatory cytokines (e.g., TNF- $\alpha$, IL-2, IL-1 $\beta$ and interferon- $\gamma$ ); these changes were at least partly mediated by inhibition of NF- $\kappa \mathrm{B}$ activation in rats (53).

In a piglet CLP model, edaravone delayed the TNF- $\alpha$ surge and prevented HMGB1 elevation, thereby maintaining MAP and prolonging survival time (61).

Effects of edaravone on ER stress. Edaravone has been shown to reduce ER stress in a study of myocarditis. Edaravone ameliorated the progression of EAM, improved LV function and decreased the ER stress signaling proteins GRP78 and caspase-12 in rats (14).

Effects of edaravone on heat shock proteins (HSPs). Anti-HSP effects of edaravone have been reported in several disease models. Edaravone reduced the levels of HSP 70 (a marker of stress) and its mRNA in an I/R-induced rat testicular torsion model (31). Edaravone also markedly reduced the expression levels of HSP 70 and its mRNA and prevented bladder dysfunction caused by AUR and subsequent catheterization in rats (60).

Effects of edaravone on tumor markers. Edaravone has been reported to reduce markers of tumorigenesis. Edaravone suppressed X-ray-induced apoptosis by inhibiting p53 in MOLT- 4 cells (22).

Effects of edaravone on cell adhesion molecules. Edaravone reduced the release of adhesion molecules, such as P-selectin, from vascular endothelial cells in rats with AMI (67). Furthermore, edaravone reduced hepatic I/R injury and hepatic E-selectin mRNA in rats (52). Histologically, edaravone reduced E-selectin immunoreactivity and neutrophil accumulation in rat hepatic sections (52). In severe hindlimb ischemia, edaravone attenuated neutrophilic infiltration, the serum level of soluble ICAM-1 and muscular edema in a rat model of myonephropathic metabolic syndrome (70).

\section{Conclusion}

The findings of research performed to date demonstrate the potential applications of edaravone for the treatment of multiple diseases, in addition to its established use in cerebral infarction. However, edaravone is currently used only to treat cerebral infarction patients in Japan. Since oxidative stress is observed in a wide variety of diseases, the abnormal generation of free radicals may underlie the etiology and aggravation of many of these diseases. Therefore, the potential therapeutic effects of edaravone in patients with various diseases should be considered. In addition to its free radical scavenging effects, edaravone has shown anti-apoptotic, anti-necrotic and anti-cytokine effects in animal models of various diseases. It is therefore important to evaluate the clinical efficacy of edaravone treatment in the diseases described above. In fact, it was recently demonstrated that edaravone is beneficial in patients with AMI (33). Clearly, further clinical studies are required to confirm the effects of edaravone observed in animal models of diseases.

\section{Acknowledgements}

The authors wish to thank Dr Rebecca Devon, Dr Stacey Tobin and Dr Nicholas D. Smith of the Edanz Group, for the editorial support.

\section{References}

1. Lapchak PA and Zivin JA: The lipophilic multifunctional antioxidant edaravone (Radicut) improves behavior following embolic strokes in rabbits: a combination therapy study with tissue plasminogen activator. Exp Neurol 215: 95-100, 2009.

2. Wang CX and Shuaib A: Neuroprotective effects of free radical scavengers in stroke. Drugs Aging 24: 537-546, 2007.

3. Green AR and Shuaib A: Therapeutic strategies for the treatment of stroke. Drug Discov Today 11: 681-693, 2006.

4. Van der Worp HB, Kappelle LJ, Algra A, Bar PR, Orgogozo JM, Ringelstein EB, Bath PM and van Gijn J: The effect of tirilazad mesylate on infarct volume of patients with acute ischemic stroke. Neurology 58: 133-135, 2002.

5. Shuaib A, Lees KR, Lyden P, Grotta J, Davalos A, Davis SM, Diener HC, Ashwood T, Wasiewski WW and Emeribe U: NXY-059 for the treatment of acute ischemic stroke. N Engl J Med 357: 562-571, 2007. 
6. Adams HP Jr, del Zoppo G, Alberts MJ, Bhatt DL, Brass L, Furlan A, Grubb RL, Higashida RT, Jauch EC, Kidwell C, Lyden PD, Morgenstern LB, Qureshi AI, Rosenwasser RH, Scott PA and Wijdicks EF: Guidelines for the early management of adults with ischemic stroke: a guideline from the American Heart Association/American Stroke Association Stroke Council, Clinical Cardiology Council, Cardiovascular Radiology and Intervention Council, and the Atherosclerotic Peripheral Vascular Disease and Quality of Care Outcomes in Research Interdisciplinary Working Groups: the American Academy of Neurology affirms the value of this guideline as an educational tool for neurologists. Stroke 38: 1655-1711, 2007.

7. Watanabe T, Yuki S, Egawa M and Nishi H: Protective effects of MCI-186 on cerebral ischemia: possible involvement of free radical scavenging and antioxidant actions. J Pharmacol Exp Ther 268: 1597-1604, 1994.

8. Yoneda Y, Uehara T, Yamasaki H, Kita Y, Tabuchi M and Mori E: Hospital-based study of the care and cost of acute ischemic stroke in Japan. Stroke 34: 718-724, 2003.

9. Yamamoto T, Yuki S, Watanabe T, Mitsuka M, Saito KI and Kogure K: Delayed neuronal death prevented by inhibition of increased hydroxyl radical formation in a transient cerebral ischemia. Brain Res 762: 240-242, 1997.

10. Lapchak PA and Araujo DM: Development of the nitrone-based spin trap agent NXY-059 to treat acute ischemic stroke. CNS Drug Rev 9: 253-262, 2003.

11. Hoehn B, Yenari MA, Sapolsky RM and Steinberg GK: Glutathione peroxidase overexpression inhibits cytochrome C release and proapoptotic mediators to protect neurons from experimental stroke. Stroke 34: 2489-2494, 2003.

12. Okatani Y, Wakatsuki A and Kaneda C: Melatonin increases activities of glutathione peroxidase and superoxide dismutase in fetal rat brain. J Pineal Res 28: 89-96, 2000.

13. Rajesh KG, Sasaguri S, Suzuki R and Maeda H: Antioxidant MCI-186 inhibits mitochondrial permeability transition pore and upregulates Bcl-2 expression. Am J Physiol Heart Circ Physiol 285: H2171-H2178, 2003.

14. Shimazaki H, Watanabe K, Veeraveedu PT, Harima M, Thandavarayan RA, Arozal W, Tachikawa H, Kodama M and Aizawa Y: The antioxidant edaravone attenuates ER-stressmediated cardiac apoptosis and dysfunction in rats with autoimmune myocarditis. Free Radic Res 44: 1082-1090, 2010.

15. Asai T, Ohno Y, Minatoguchi S, Funaguchi N, Yuhgetsu H, Sawada M, Takemura G, Komada A, Fujiwara T and Fujiwara H: The specific free radical scavenger edaravone suppresses bleomycin-induced acute pulmonary injury in rabbits. Clin Exp Pharmacol Physiol 34: 22-26, 2007.

16. Ninomiya M, Shimada M, Harada N, Shiotani S, Hiroshige S Soejima Y, Suehiro T and Sugimachi K: Beneficial effect of MCI-186 on hepatic warm ischemia-reperfusion in the rat. Transplantation 74: 1470-1472, 2002.

17. Nakamoto N, Tada S, Kameyama K, Kitamura K, Kurita S, Saito Y, Saito H and Ishii H: A free radical scavenger, edaravone, attenuates steatosis and cell death via reducing inflammatory cytokine production in rat acute liver injury. Free Radic Res 37: 849-859, 2003.

18. Tada S, Nakamoto N, Kameyama K, Tsunematsu S, Kumagai N, Saito $\mathrm{H}$ and Ishii $\mathrm{H}$ : Clinical usefulness of edaravone for acute liver injury. J Gastroenterol Hepatol 18: 851-857, 2003.

19. Miyasou T, Kwon AH, Tsuji K, Qiu Z, Okumura T and Kamiyama Y: Edaravone prevents Fas-induced fulminant hepatic failure in mice by regulating mitochondrial Bcl-xL and Bax. Shock 30: 212-216, 2008

20. Nagatani S, Sudo T, Murakami Y, Uemura K, Hiyama E and Sueda T: Edaravone, a free radical scavenger, promotes engraftment of intraportally transplanted islet cells. Pancreas 40: 126-130, 2011

21. Satoh M, Kashihara N, Fujimoto S, Horike H, Tokura T, Namikoshi T, Sasaki $\mathrm{T}$ and Makino $\mathrm{H}$ : A novel free radical scavenger, edarabone, protects against cisplatin-induced acute renal damage in vitro and in vivo. J Pharmacol Exp Ther 305: 1183-1190, 2003

22. Sasano N,Enomoto A,Hosoi Y,Katsumura Y, Matsumoto Y, Morita A, Shiraishi K, Miyagawa K, Igaki $\mathrm{H}$ and Nakagawa K: Edaravone, a known free radical scavenger, enhances X-ray-induced apoptosis at low concentrations. Cancer Lett 293: 52-57, 2010.

23. Sasano N, Enomoto A, Hosoi Y, Katsumura Y, Matsumoto Y, Shiraishi K, Miyagawa K, Igaki H and Nakagawa K: Free radical scavenger edaravone suppresses X-ray-induced apoptosis through p53 inhibition in MOLT-4 cells. J Radiat Res 48: 495-503, 2007.
24. Minhaz U, Tanaka M, Tsukamoto H, Watanabe K, Koide S, Shohtsu A and Nakazawa H: Effect of MCI-186 on postischemic reperfusion injury in isolated rat heart. Free Radic Res 24: 361-367, 1996.

25. Wu TW, Zeng LH, Wu J and Fung KP: Myocardial protection of MCI-186 in rabbit ischemia-reperfusion. Life Sci 71: 2249-2255, 2002.

26. Ninomiya M, Shimada M, Harada N, Soejima Y, Suehiro T and Maehara Y: The hydroxyl radical scavenger MCI-186 protects the liver from experimental cold ischaemia-reperfusion injury. Br J Surg 91: 184-190, 2004

27. Suzuki F, Hashikura Y, Ise H, Ishida A, Nakayama J, Takahashi M, Miyagawa S and Ikeda U: MCI-186 (edaravone), a free radical scavenger, attenuates hepatic warm ischemia-reperfusion injury in rats. Transpl Int 18: 844-853, 2005.

28. Yang T, Mao YF, Liu SQ, Hou J, Cai ZY, Hu JY, Ni X, Deng XM and Zhu XY: Protective effects of the free radical scavenger edaravone on acute pancreatitis-associated lung injury. Eur J Pharmacol 630: 152-157, 2010

29. Sueishi K, Mishima K, Makino K, Itoh Y, Tsuruya K, Hirakata H and Oishi R: Protection by a radical scavenger edaravone against cisplatin-induced nephrotoxicity in rats. Eur J Pharmacol 451: 203-208, 2002

30. Doi K, Suzuki Y,Nakao A,Fujita T and Noiri E: Radical scavenger edaravone developed for clinical use ameliorates ischemia/reperfusion injury in rat kidney. Kidney Int 65: 1714-1723, 2004.

31. Tamamura M, Saito M, Kinoshita Y, Shimizu S, Satoh I, Shomori K, Dimitriadis F and Satoh K: Protective effect of edaravone, a free-radical scavenger, on ischaemia-reperfusion injury in the rat testis. BJU Int 105: 870-876, 2010

32. Tsujita K, Shimomura H, Kawano H, Hokamaki J, Fukuda M, Yamashita T, Hida S, Nakamura Y, Nagayoshi Y, Sakamoto T, Yoshimura M, Arai $\mathrm{H}$ and Ogawa $\mathrm{H}$ : Effects of edaravone on reperfusion injury in patients with acute myocardial infarction. Am J Cardiol 94: 481-484, 2004

33. Tsujita K, Shimomura H, Kaikita K, Kawano H, Hokamaki J, Nagayoshi Y, Yamashita T, Fukuda M, Nakamura Y, Sakamoto T, Yoshimura $\mathrm{M}$ and Ogawa $\mathrm{H}$ : Long-term efficacy of edaravone in patients with acute myocardial infarction. Circ J 70: 832-837, 2006.

34. Sukmawan R, Yada T, Toyota E, Neishi Y, Kume T, Shinozaki Y, Mori H, Ogasawara Y, Kajiya F and Yoshida K: Edaravone preserves coronary microvascular endothelial function after ischemia/reperfusion on the beating canine heart in vivo. J Pharmacol Sci 104: 341-348, 2007.

35. Onogi H, Minatoguchi S, Chen XH, Bao N, Kobayashi H, Misao Y, Yasuda S, Yamaki T, Maruyama R, Uno Y, Arai M, Takemura $\mathrm{G}$ and Fujiwara $\mathrm{H}$ : Edaravone reduces myocardial infarct size and improves cardiac function and remodelling in rabbits. Clin Exp Pharmacol Physiol 33: 1035-1041, 2006.

36. Yagi $\mathrm{H}$, Horinaka $\mathrm{S}$ and Matsuoka $\mathrm{H}$ : Edaravone prevented deteriorated cardiac function after myocardial ischemiareperfusion via inhibiting lipid peroxidation in rat. J Cardiovasc Pharmacol 46: 46-51, 2005

37. Yamazaki K, Miwa S, Toyokuni S, Nemoto S, Oriyanhan W, Takaba K, Saji Y, Marui A, Nishina T, Ikeda T and Komeda M: Effect of edaravone, a novel free radical scavenger, supplemented to cardioplegia on myocardial function after cardioplegic arrest: in vitro study of isolated rat heart. Heart Vessels 24: 228-235, 2009.

38. Nimata M, Okabe TA, Hattori M, Yuan Z, Shioji K and Kishimoto C: MCI-186 (edaravone), a novel free radical scavenger, protects against acute autoimmune myocarditis in rats. Am J Physiol Heart Circ Physiol 289: H2514-H2518, 2005.

39. Kotani Y, Ishino K, Osaki S, Honjo O, Suezawa T, Kanki K, Yutani C and Sano S: Efficacy of MCI-186, a free-radical scavenger and antioxidant, for resuscitation of nonbeating donor hearts. J Thorac Cardiovasc Surg 133: 1626-1632, 2007.

40. Yamawaki M, Sasaki N, Shimoyama M, Miake J, Ogino K, Igawa O, Tajima F, Shigemasa C and Hisatome I: Protective effect of edaravone against hypoxia-reoxygenation injury in rabbit cardiomyocytes. Br J Pharmacol 142: 618-626, 2004.

41. Akao T, Takeyoshi I, Totsuka O, Arakawa K, Muraoka M, Kobayashi K, Konno K, Matsumoto K and Morishita Y: Effect of the free radical scavenger MCI-186 on pulmonary ischemia-reperfusion injury in dogs. J Heart Lung Transplant 25: 965-971, 2006.

42. Qiu W, Gu H, Zheng L, Zhou J, Chen D and Chen Y: Pretreatment with edaravone reduces lung mitochondrial damage in an infant rabbit ischemia-reperfusion model. J Pediatr Surg 43: 2053-2060, 2008 . 
43. Reyes YA, Shimoyama T, Akamatsu $\mathrm{H}$ and Sunamori $\mathrm{M}$ : MCI-186 (edaravone), a free radical scavenger, attenuates ischemia-reperfusion injury and activation of phospholipase $\mathrm{A}(2)$ in an isolated rat lung model after $18 \mathrm{~h}$ of cold preservation. Eur J Cardiothorac Surg 29: 304-311, 2006.

44. Tajima S, Bando M, Ishii Y, Hosono T, Yamasawa H, Ohno S, Takada T, Suzuki E, Gejyo F and Sugiyama Y: Effects of edaravone, a free-radical scavenger, on bleomycin-induced lung injury in mice. Eur Respir J 32: 1337-1343, 2008.

45. Tomatsuri N, Yoshida N, Takagi T, Katada K, Isozaki Y, Imamoto E, Uchiyama K, Kokura S, Ichikawa H, Naito Y, Okanoue $\mathrm{T}$ and Yoshikawa T: Edaravone, a newly developed radical scavenger, protects against ischemia-reperfusion injury of the small intestine in rats. Int J Mol Med 13: 105-109, 2004.

46. Shimizu K, Koga H, Iida M and Haruma K: Microcirculatory changes in experimental mesenteric longitudinal ulcers of the small intestine in rats. Dig Dis Sci 52: 3019-3028, 2007.

47. Kono H, Asakawa M, Fujii H, Maki A, Amemiya H, Yamamoto M, Matsuda M and Matsumoto Y: Edaravone, a nove free radical scavenger, prevents liver injury and mortality in rats administered endotoxin. J Pharmacol Exp Ther 307: 74-82, 2003

48. Hiranuma S, Ito K, Noda Y, Ozasa H, Koike Y and Horikawa S Amelioration of hepatic ischemia/reperfusion injury in the remnant liver after partial hepatectomy in rats. J Gastroenterol Hepatol 22: 2167-2172, 2007.

49. Kono H, Woods CG, Maki A, Connor HD, Mason RP, Rusyn I and Fujii $\mathrm{H}$ : Electron spin resonance and spin trapping technique provide direct evidence that edaravone prevents acute ischemiareperfusion injury of the liver by limiting free radical-mediated tissue damage. Free Radic Res 40: 579-588, 2006.

50. Nakamura A, Akamatsu Y, Miyagi S, Fukumori T, Sekiguchi S and Satomi S: A free radical scavenger, edaravone, prevents ischemia-reperfusion injury in liver grafts from non-heartbeating donors. Transplant Proc 40: 2171-2174, 2008.

51. Okatani Y, Wakatsuki A, Enzan H and Miyahara Y: Edaravone protects against ischemia/reperfusion-induced oxidative damage to mitochondria in rat liver. Eur J Pharmacol 465: 163-170, 2003.

52. Taniguchi M, Uchinami M, Doi K, Yoshida M, Sasaki H, Tamagawa K, Horiuchi $\mathrm{T}$ and Tanaka K: Edaravone reduces ischemia-reperfusion injury mediators in rat liver. J Surg Res 137: 69-74, 2007.

53. Tsuji K, Kwon AH, Yoshida H, Qiu Z, Kaibori M, Okumura T and Kamiyama Y: Free radical scavenger (edaravone) prevents endotoxin-induced liver injury after partial hepatectomy in rats. J Hepatol 42: 94-101, 2005.

54. Yoshida H, Kwon AH, Habara K, Yamada M, Kaibori M, Kamiyama Y, Nishizawa M, Ito $\mathrm{S}$ and Okumura T: Edaravone inhibits the induction of iNOS gene expression at transcriptional and posttranscriptional steps in murine macrophages. Shock 30 : 734-739, 2008

55. Araki, Y, Andoh A, Yokono T, Asano N, Yoshikawa K, Bamba S, Ishizuka I and Fujiyama Y: The free radical scavenger edaravone suppresses experimental closed duodenal loop-induced acute pancreatitis in rats. Int J Mol Med 12: 121-124, 2003.

56. Tahara M, Nakayama M, Jin MB, Fujita M, Suzuki T, Taniguchi M, Shimamura T, Furukawa $\mathrm{H}$ and Todo $\mathrm{S}$ : A radical scavenger, edaravone, protects canine kidneys from ischemiareperfusion injury after 72 hours of cold preservation and autotransplantation. Transplantation 80: 213-221, 2005.

57. Someya T, Kaneko K, Yamada T and Yamashiro Y: Effect of a novel free radical scavenger, edaravone, on puromycin aminonucleoside induced nephrosis in rats. Pediatr Nephrol 20: $1430-1434,2005$.
58. Matsumura H, Ashida A, Hirano K, Nakakura $\mathrm{H}$ and Tamai $\mathrm{H}$ : Protective effect of radical scavenger edaravone against puromycin nephrosis. Clin Nephrol 66: 405-410, 2006.

59. Matsumoto S, Hanai T, Yoshioka N, Shimizu N, Sugiyama T, Uemura H and Levin RM: Edaravone protects against ischemia/ reperfusion-induced functional and biochemical changes in rat urinary bladder. Urology 66: 892-896, 2005.

60. Shimizu S, Saito M, Kinoshita Y, Kazuyama E, Tamamura M, Satoh I and Satoh K: Acute urinary retention and subsequent catheterization cause lipid peroxidation and oxidative DNA damage in the bladder: preventive effect of edaravone, a freeradical scavenger. BJU Int 104: 713-717, 2009.

61. Kato S, Hussein MH, Kakita H, Goto T, Daoud GA, Kato T, Sugiura T, Nobata M, Nakajima Y, Endo T, Mizuno K, Ito T, Kato I, Suzuki S and Togari H: Edaravone, a novel free radical scavenger, reduces high-mobility group box 1 and prolongs survival in a neonatal sepsis model. Shock 32: 586-592, 2009.

62. Koizumi T, Tanaka H, Sakaki S and Shimazaki S: The therapeutic efficacy of edaravone in extensively burned rats. Arch Surg 141: 992-995, 2006.

63. Mori T, Yamamoto H, Tabata T, Shimizu T, Endo Y, Hanasawa K, Fujimiya M and Tani T: A free radical scavenger, edaravone (MCI-186), diminishes intestinal neutrophil lipid peroxidation and bacterial translocation in a rat hemorrhagic shock model. Crit Care Med 33: 1064-1069, 2005.

64. Uji Y, Yamamoto $\mathrm{H}$, Mori $\mathrm{T}$, Akabori $\mathrm{H}$, Tsuchihashi $\mathrm{H}$, Shimizu T, Endo Y and Tani T: Edaravone improves the survival of rats subjected to hemorrhagic shock without resuscitation. Surg Today 38: 476-477, 2008

65. Kawasaki T, Ishihara K, Ago Y, Nakamura S, Itoh S, Baba A and Matsuda T: Protective effect of the radical scavenger edaravone against methamphetamine-induced dopaminergic neurotoxicity in mouse striatum. Eur J Pharmacol 542: 92-99, 2006.

66. Nakamura Y, Yamada Y, Shimomura H, Nagayoshi Y, Tsujita K, Yamashita T, Fukuda M, Ohba K, Nako H, Ogura Y, Chitose T, Yamaguchi M, Nagata T, Soejima H, Kaikita K, Sugiyama S and Ogawa H: The effect of edaravone on plasma monocyte chemoattractant protein-1 levels in patients with acute myocardial infarction. J Cardiol 54: 416-424, 2009.

67. Onimaru S, Nakamura K, Kariyazono H, Ikeda R, Ueno T, Fukumoto Y, Yabuki A, Sakata R and Yamada K: Inhibitory effects of edaravone on the production of tumor necrosis factoralpha in the isolated heart undergoing ischemia and reperfusion. Heart Vessels 21: 108-115, 2006.

68. Okabe TA, Kishimoto C, Hattori M, Nimata M, Shioji K and Kita T: Cardioprotective effects of 3-methyl-1-phenyl-2-pyrazolin-5-one (MCI-186), a novel free radical scavenger, on acute autoimmune myocarditis in rats. Exp Clin Cardiol 9: 177-180, 2004.

69. Tajima S, Soda M, Bando M, Enomoto M, Yamasawa H, Ohno S, Takada T, Suzuki E, Gejyo F and Sugiyama Y: Preventive effects of edaravone, a free radical scavenger, on lipopolysaccharideinduced lung injury in mice. Respirology 13: 646-653, 2008.

70. Kaneko K, Yonemitsu Y, Fujii T, Onimaru M, Jin CH, Inoue M, Hasegawa M, Onohara T, Maehara Y and Sueishi K: A free radical scavenger but not FGF-2-mediated angiogenic therapy rescues myonephropathic metabolic syndrome in severe hindlimb ischemia. Am J Physiol Heart Circ Physiol 290: H1484-H1492, 2006. 